\title{
Efecto del hongo entomopatógeno Beauveria bassiana (balsamo) Vuillemin en el control de la oveja ked (Melophagus ovinus)
}

\author{
Effect of entomopathogenic fungus Beauveria bassiana (balsamo) Vuillemin on \\ the control of sheep ked (Melophagus ovinus)
}

\author{
Adrián Hernando Valbuena Puentes ${ }^{1}$, Adriana Marcela Galindo Soracá ${ }^{2,3}$, \\ Yefer Mauricio Boyacá Quintana²
}

\section{Resumen}

Melophagus ovinus (oveja ked) es un parásito común del ovino, su control se basa en tratamientos químicos; sin embargo, las consecuencias de tipo ambiental y en la salud pública obliga a la aplicación de tratamientos alternativos como los hongos entomopatógenos. El estudio tuvo como objetivo determinar el efecto del hongo Beuveria bassiana sobre el parásito en ovinos naturalmente infestados. Se utilizaron 15 ovejas adultas Hampshire cruzadas, distribuidas al azar en tres tratamientos de aspersión con diluciones: T1: $3.2 \times 10^{8}$ conidios $/ \mathrm{ml}, \mathrm{T} 2: 3.2 \times 10^{8}$ conidios $/ \mathrm{ml}+5 \%$ de azúcar y T control: Agua destilada. Se realizó el conteo diario de los parásitos sobre el animal para determinar la densidad poblacional y la eficacia de los tratamientos. La densidad poblacional fue controlada a partir del día 14 con $B$. bassiana, reduciendo la presencia de la ked en un $90 \%$ (T1 y T2), frente a un $5.9 \%$ en el T Control $(\mathrm{p}<0.05)$. El estudio sugiere que $B$. bassiana funciona con una eficacia de $89.3 \%$ para el control de M. ovinus

Palabras clave: control biológico, falsa garrapata, ovino, eficacia, in vivo

\section{AbSTRaCt}

Melophagus ovinus (sheep ked) is a common parasite of sheep, its control is based on chemical treatments; however, the environmental and public health consequences require the application of alternative treatments such as entomopathogenic fungi. The

\footnotetext{
${ }^{1}$ Programa Medicina Veterinaria, Facultad de Ciencias Agrarias y Ambientales, Fundación Universitaria Juan de Castellanos, Tunja, Boyacá, Colombia

2. Facultad de Ciencias Agrarias y Ambientales, Fundación Universitaria Juan de Castellanos, Tunja, Boyacá, Colombia

${ }^{3}$ E-mail: agalindo@jdc.edu.co
}

Recibido: 23 de julio de 2020

Aceptado para publicación: 28 de enero de 2021

Publicado: 24 de abril de 2021 
study aimed to determine the effect of the fungus Beuveria bassiana on the parasite in naturally infested sheep. Fifteen adult Hampshire crossbred sheep were used, randomly

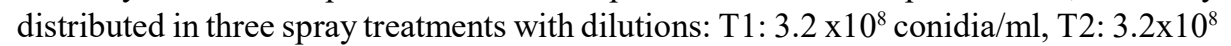
conidia/ml $+5 \%$ sugar and control $\mathrm{T}$ : distilled water. The daily parasites were counted on the animal to determine the population density and the efficacy of the treatments. The population density was controlled from day 14 with $B$. bassiana, reducing the presence of ked by $90 \%$ (T1 and T2), compared to $5.9 \%$ in the T Control ( $<<0.05)$. The study suggests that B. bassiana works with an efficacy of $89.3 \%$ for the control of M. ovinus.

Key words: biological control, false tick, sheep, efficacy, in vivo

\section{INTRODUCCIÓN}

El Melophagus ovinus (M. ovinus) también llamado oveja ked es un díptero hematófago sin alas de la familia Hippoboscidae que se ubica en la lana de las ovejas. Es un parásito que puede llegar a causar anemia en casos de alta carga parasitaria, afectar la conducta de pastoreo por la irritación y el prurito, pérdidas en la ganancia de peso diaria y en la producción de lana y piel (Antonelli y Sandoval, 2010; Larroza et al., 2012; Larroza, 2013; Duan et al., 2017).

El tratamiento contra esta parasitosis se realiza con productos inyectables como lactonas macrocíclicas, así como con baños de inmersión y aspersión con cipermetrinas (Álvarez, 2007); sin embargo, el uso de tratamientos de control alternos con hongos, diatomeas y nemátodos entomopatógenos ha sido considerado (Gómez et al., 2012).

Los hongos entomopatógenos son enemigos naturales de los insectos; y pueden llegar a exterminar poblaciones de plagas sin generar efectos perjudiciales para la salud humana o el medio ambiente (Khan et al., 2012). Se conoce que hongos del género Beauveria son eficaces para el control de varios insectos por su alta patogenicidad al penetrar en el hemocele de su hospedero y causarle la muerte tras la producción de toxinas (Mejía et al., 2016; Rao y Narladkar, 2018). Las esporas del hongo entran en contacto con la superficie quitinosa del insecto, donde inicia el mecanismo entomopatógeno de adhesión, germinación y penetración por medio de enzimas proteasas, quitinasas y lipasas (Monzón, 2001; Pedrini et al., 2007; Wang y Leger, 2007) y dentro del huésped contrarresta los mecanismos inmunes del insecto propiciando efectos nocivos y la muerte (Téllez et al., 2009).

El presente trabajo tuvo como objetivo evaluar el efecto in vivo del hongo entomopatógeno $B$. bassiana en el parásito $M$. ovinus de ovejas naturalmente infestadas provenientes del municipio de Güicán de la Sierra, Boyacá, Colombia.

\section{Materiales y Métodos}

\section{Lugar del Estudio}

El estudio se realizó en la finca La Cabaña, ubicada en el municipio de Güicán de la Sierra, departamento de Boyacá, Colombi. La región se encuentra a una altitud de $2895 \mathrm{msnm}$, y presenta una temperatura media de $13{ }^{\circ} \mathrm{C}$ y humedad relativa de 80\% (Alcaldía Guicán de la Sierra, 2019). 


\section{Animales}

Se utilizaron 15 ovejas adultas Hampshire Down cruzadas que se encontraban naturalmente infestadas por M. ovinus. Los animales tenían un peso promedio de 40 $\mathrm{kg}$, edades de 2.5 a 3 años y eran parte del rebaño comercial de la finca. Las ovejas se distribuyeron en tres grupos en corrales de $12 \mathrm{~m}^{2}$, con periodos de pastoreo de 4 horas al día en praderas de pasto kikuyo (Pennisetum clandestinum) y agua a voluntad.

\section{Hongo Entomopatógeno}

Se usó la cepa comercial del hongo Beuveria bassiana ADRAL ${ }^{\circledR} w p$ de BioCrop (Colombia), en la presentación líquida en agua destilada con Tween 80 al $0.1 \%$. Se tomó $1 \mathrm{ml}$ en una cámara de Neubauer y se calculó el número total de conidios por mililitro usando la fórmula (Cañedo y Ames, 2004) Conidias $(\mathrm{ml})=\mathrm{N} .^{\circ}$ conidias contadas $\mathrm{x}$ $25000 \mathrm{x}$ factor de dilución, obteniéndose una concentración de $2 \times 10^{8}$ conidios $/ \mathrm{ml}$ de $B$. bassiana.

Adicionalmente se cultivó el hongo en caja Petri con agar Sabouraud usando la técnica de estría por agotamiento en incubación a $30{ }^{\circ} \mathrm{C}$ y $80 \%$ HR durante $72 \mathrm{~h}$. La identificación del hongo se hizo con tinción de lactofenol a interpretación microscópica de las características morfológicas según el Manual de Hongos Entomopatógenos (Cañedo y Ames, 2004).

\section{Tratamientos}

Se trabajó con tres tratamientos:

- T1: $8.3 \mathrm{ml}$ de la cepa comercial en $500 \mathrm{ml}$ de agua destilada $\left(3.2 \times 10^{6}\right.$ conidias $\left./ \mathrm{ml}\right)$

- T2: $8.3 \mathrm{ml}$ de la cepa comercial en $500 \mathrm{ml}$ de agua destilada y azucara al 5\% como coadyuvante $\left(3.2 \times 10^{6}\right.$ conidias $\left./ \mathrm{ml}\right)$

- T(Control): $500 \mathrm{ml}$ de agua destilada
Cuadro 1. Tablas de frecuencia de grados de densidad poblacional

\begin{tabular}{cc}
\hline Grado & $\begin{array}{c}\text { Parásitos } \\
(\mathrm{n})\end{array}$ \\
\hline 0 & 0 \\
1 & $1-12$ \\
2 & $13-24$ \\
3 & $25-36$ \\
4 & $37-50$ \\
\hline
\end{tabular}

Los tratamientos fueron aplicados una vez por aspersión con bomba de espalda a cada animal cubriendo toda la superficie corporal del animal. La aplicación se hizo en horas de la tarde con los animales dentro del aprisco para evitar efectos de la luz solar sobre la cepa fúngica.

\section{Identificación de Parásitos}

El conteo de los parásitos se realizó cada 24 horas a la misma hora en la mañana de forma directa y durante tres semanas. Se observó cada canal formado naturalmente por el vellón desde cuello, pecho, brazo y antebrazo de la región lateral izquierda, repitiendo la observación en la región contralateral (Becerra y Otrando, 2020; Small, 2005). Los datos se organizaron en tablas de frecuencia con intervalos, a los que para efectos de este estudio se les denominaron grado de densidad poblacional (Cuadro 1).

Para determinar el efecto del hongo se realizó conteo diario de los parásitos presentes en área corporal establecida. El valor fue expresado en porcentaje con el número de melófagos vivos en cada repetición y tratamiento. Los valores se reemplazaron con las siguientes fórmulas de eficacia de ectoparasiticidas (Abbot, 1925; Holdsworth et al., 2006): 


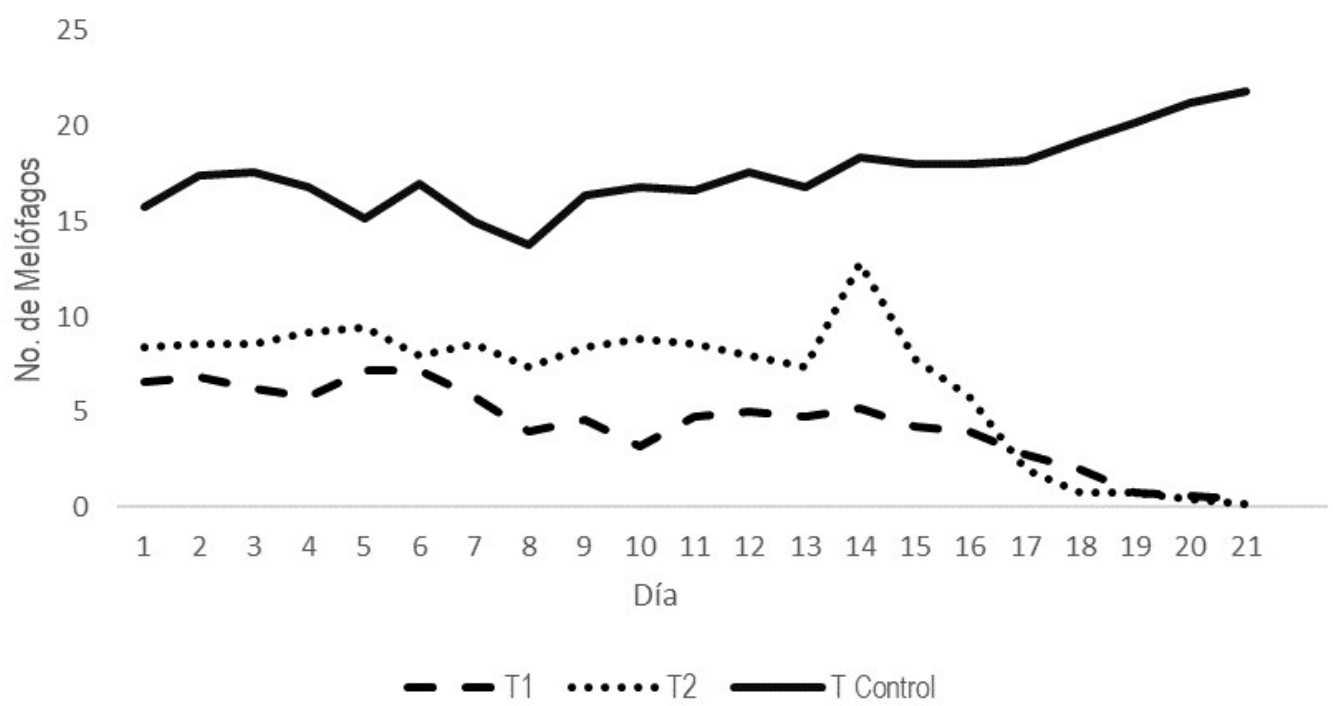

Figura 1. Número de Melophagus ovinus en cuello, pecho, brazo y antebrazo de la región lateral izquierda y derecha de ovinos tratados con Beuveria bassiana. T1: $3.2 \times 10^{6}$ conidias/ml de agua destilada; T2; como $\mathrm{T} 1$ pero con agua destilada azucarada al 5\%; T Control: agua destilada
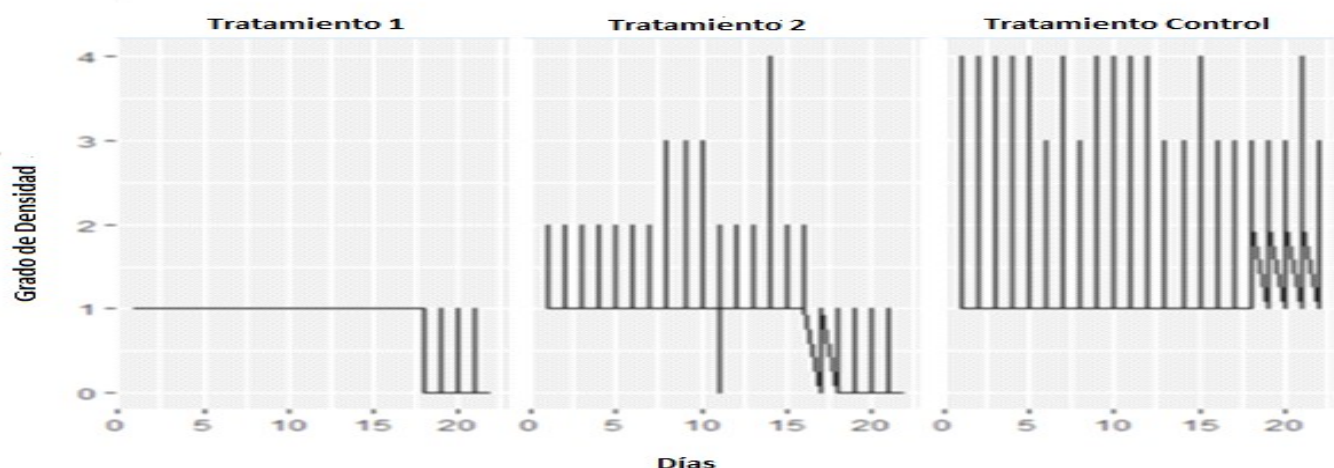

Figura 2. Densidad poblacional de Melophagus ovinus tratados con Beuveria bassiana (LSD de Fisher). T1: $3.2 \times 10^{6}$ conidias/ml de agua destilada; T2; como T1 pero con agua destilada azucarada al 5\%; T Control: agua destilada

- \% Supervivencia $=($ Número de melógagos vivos / número total de melófagos) * 100

- $\%$ Mortalidad $(\%)=100$ - Supervivencia $(\%)$
- \% Eficacia $=[(\%$ Supervivencia del Control - \% Supervivencia del tratamiento) / (\% Supervivencia del Control] $* 100$ 

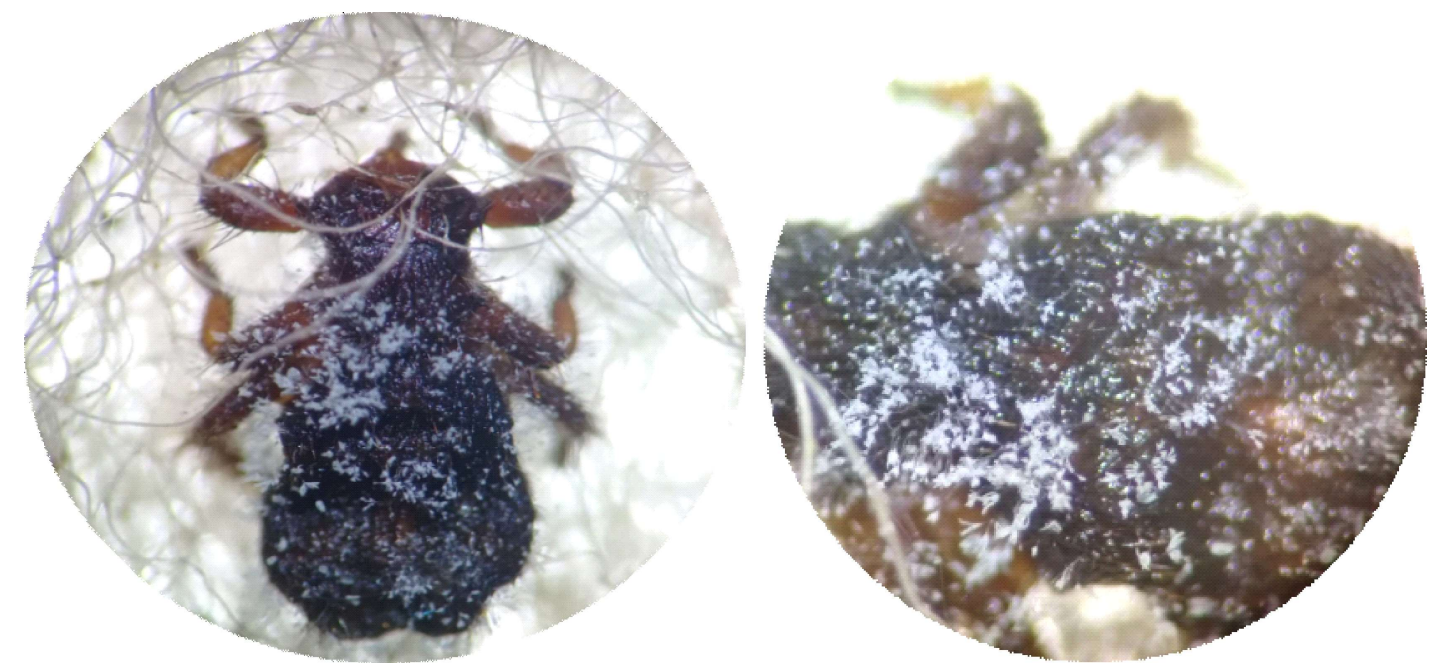

Figura 3. Crecimiento de micelios sobre cutícula de Melophagus ovinus tratados con Beuveria bassiana

Los resultados fueron analizados mediante análisis de varianza y las diferencias entre medias mediante la prueba de Fisher $(\mathrm{p}<0.05)$.

\section{Resultados}

El número promedio de melófagos al inicio del estudio fue de $4.3 \pm 2.1,6.6 \pm 3.5 \mathrm{y}$ de $17.5 \pm 1.9$ para $\mathrm{T} 1, \mathrm{~T} 2$ y $\mathrm{T}($ Control), respectivamente. La variabilidad en el número de M. ovinos en cada conteo pudo asociarse al desplazamiento de parásitos dentro del área de conteo (Figura 1).

La población tratada con $B$. bassiana se redujo en 50\% a partir del día 16 del estudio (Figura 1). Los ovinos de T1 presentaron valores de control más favorables, que fueron igualados por los ovinos de T2 a los 21 días llegando a un promedio de 0.5 parásitos por conteo, valor significativamente menor que en el grupo control $(\mathrm{p}<0.05)$.
Los tratamientos con B. bassiana tuvieron densidad «0» a partir de la segunda semana, mientras que la densidad en el grupo control fue de $« 4 »(p<005)$ (Figura 2$)$. La mortalidad fue determinada a partir de la relación de supervivencia, ya que el conteo de los insectos muertos que caen del vellón del animal fue imperceptible. En los parásitos muertos o moribundos que fueron colectados se evidenció una coloración más oscura, nulas (o poca) respuesta al estímulo y algunos de ellos con micelios en la cutícula (Figura 3).

Los porcentajes de mortalidad de $M$. ovinus en los días 7, 14 y 21 del estudio se muestran en el Cuadro 2. La mortalidad se incrementó notoriamente en T1 y T2, llegando a ser de $90 \%$ en el día 21 , en tanto que en el grupo control fue de $5.9 \%(\mathrm{p}<0.05)$. La eficacia en el control de M. ovinus fue de $89.3 \%(\mathrm{p}<0.05)$, indicando la conveniencia de los tratamientos con hongos (Holdsworth et al., 2006). 
Cuadro 2. Porcentaje de mortalidad en el día 7, 14 y 21 de Melophagus ovinus en ovinos tratados con Beuveria bassiana

\begin{tabular}{cccc}
\hline Día & \multicolumn{3}{c}{ Mortalidad $(\%)$} \\
\cline { 2 - 4 } & T1 & T2 & T control \\
\cline { 2 - 4 } 7 & $18.3^{\mathrm{a}}$ & $6.0^{\mathrm{b}}$ & $7.4^{\mathrm{b}}$ \\
14 & $18.8^{\mathrm{a}}$ & $11.0^{\mathrm{b}}$ & $5.9^{\mathrm{c}}$ \\
21 & $90.0^{\mathrm{a}}$ & $90.0^{\mathrm{a}}$ & $5.9^{\mathrm{b}}$ \\
\hline
\end{tabular}

$a, b, c$ Valores promedios de filas con diferentes letra son diferentes $(p<0.05)$

$\mathrm{T} 1: 3.2 \times 10^{6}$ conidias/ml de agua destilada; T2; como T1 pero con agua destilada azucarada al 5\%; T Control: agua destilada

\section{Discusión}

Las características biológicas de $M$. ovinus y su notable necesidad de realizar todo su ciclo biológico en la oveja, su hospedero natural, contribuyen a que su cultivo in vitro se dificulte, ya que solo sobrevive una media de 24 horas fuera del hospedero (Manosalva et al., 2013).

A pesar de que los tratamientos se unificaron por peso y edad, se encontraron diferencias en la densidad poblacional inicial, lo cual puede atribuirse a factores de susceptibilidad intrínsecos como condición corporal, densidad de la lana, edad, gestación, y sexo que permiten una mayor o menor colonización de los melófagos (Olaechea et al., 2006; Olaechea, 2009; Scasta y Koepke, 2016). La población de melófagos se mantuvo muy activa durante el estudio, desplazándose en el vellón, próximos o alejados de la piel, comportamiento motivado por variaciones de temperatura corporal y ambiental (Olaechea, 2009).

Existen diversas causas naturales que generan bajas en la población del melófago, como adaptabilidad y resistencia propia del hospedador (Nelson y Bainborough, 1963;
Nelson y Kozub, 1980), aunque ha sido sugerido que los parásitos ubicados en la superficie del vellón tienen mayor riesgo de mortalidad por caer al suelo, por depredación e incluso por ingestión por parte de la oveja (Becerra y Otrando, 2020); sin embargo, la densidad poblacional «0» obtenida en los tratamientos con $B$ bassiana indican un efecto controlador del hongo para M. ovinus al comparar las densidades con la del grupo no tratado.

La mortalidad obtenida en T1 y T2 de $90 \%$ fue superior a la reportada por Gómez et al. (2012) con mortalidades de $64.1 \%$ en ovejas con vellón largo y de $79.4 \%$ en ovinos esquilados, donde estos grupos fueron tratados con formulación líquida de $10^{9}$ conidios $/ \mathrm{ml} \mathrm{de}$ B. bassiana por 30 días. De otra parte, el $89 \%$ de eficacia de los tratamientos con $B$. bassiana, se acercan a la eficacia reportada por Tofiño et al. (2018) de 96.8\%, al aplicar aspersiones del hongo a una concentración de $10^{6}$ conidias/ml para el control de garrapatas $R$. microplus. Las diferencias pueden sugerir que los datos de mortalidad varían en virtud de la concentración, el potencial patógeno de la cepa fúngica y las condiciones de humedad y temperatura adecuadas para el crecimiento de B. bassiana. 
Los M. ovinos de los grupos tratados presentaron disminución de movimiento hasta parálisis y cambio en la coloración tornándose a un café oscuro. Los cambios de color pueden estar asociados a la melanización, proceso que es descrito en los insectos como una respuesta del sistema inmune humoral a la llegada de cualquier agente externo, y se conoce bajo el nombre de sistema profenoloxidasa (Vargas y Ortega, 1994; Chase et al., 2000; Chávez et al., 2014; Jiang et al., 2019).

El efecto del hongo entomopatógeno fue comprobado. La muerte de los parásitos contrasta con su capacidad patógena, que se resume en ingresar a la hemolinfa ocasionando disrupción en la homeodinamia parasitaria y con ello la muerte (Kirkland et al., 2005; Pucheta et al., 2006; Harrison y Bonning, 2010; Ortiz y Nemat, 2013). La muerte del parásito y el tiempo de presentación del hongo influenciada por la actividad enzimática y la cantidad de sustrato establece que entre 3 a 7 días de la muerte del parásito ocurre la esporulación fuera del esqueleto (Angelo et al., 2010). La esporulación puede ocurrir interna y externamente, dependiendo de la humedad, que cuando es baja se dificulta la esporulación externa pudiendo ser el caso de los tratamientos (Delgado y Murcia, 2011); sin embargo, este comportamiento justifica que una baja germinación puede igual generar la muerte de los parásitos (Feijó et al., 2007), pues los conidios pudieron producir toxinas que generaron la patogenicidad. La muerte de los parásitos no se encontró asociada a la presentación de micelios de $B$. bassiana. La producción de micelios no está asociada a mayor virulencia y a la patogenicidad (Obando y Bustillo, 2011).

La aplicación de azúcar como coadyudante del B. bassiana en el presente estudio no alteró la eficacia entre los tratamientos, no evidenció el objetivo de su aplicación para mejorar la eficacia en campo del producto, la estabilidad durante su aplicación, ni la adhesión y penetración del hongo en el parásito (Bastidas et al 2009), datos que difieren a
Arrubla et al. (2008), quienes mezclaron $B$. bassiana con azúcar al 5\% para el control de la broca, demostrando un mejor resultado.

\section{Conclusiones}

- La aspersión de Beauveria bassiana a concentración de $3.32 \times 10^{6}$ conidias $/ \mathrm{ml}$ generó un $89 \%$ de control de la población de Melophagus ovinus a los 21 días del tratamiento.

- La mortalidad y la eficacia del tratamiento se atribuye a los mecanismos patógenos propios del hongo $B$. bassiana.

\section{Agradecimientos}

Los autores agradecen a la Fundación Universitaria Juan de Castellanos y su Facultad de Ciencias Agrarias y Ambientales por apoyar el proyecto de investigación.

\section{Literatura Citada}

1 Abbott WS. 1987. A method of computing the effectiveness of an insecticide 1925. $\mathrm{j}$ am mosquito contr 18 : 265-267.

2 Alcaldía Güicán de la Sierra 2019. Nuestro municipio. [Internet]. Disponible en http://www.guican-boyaca.gov.co.

3 Angelo I, Gôlo P, Camargo M, Kluck G, Folly E, Bittencourt VR. 2010. Haemolymph protein and lipid profile of Rhipicephalus (Boophilus) microplus infected by fungi. Transbound Emerg Dis 57: 79-83. doi: $10.1111 / \mathrm{j} .18651682$.2010.01119.x

4 Antonelli A, Sandoval L. 2010. Control of sheep keds. Washington State University. [Internet]. Available in: https:/ /research.libraries.wsu.edu/xmlui/ handle/2376/16184

5 Alvarez F. 2007. Eficacia de cuatro antiparasitarios para el control de Melophagus ovinus en la Región de Magallanes, Patagonia Chilena. Parasitol 
Latinoam 62: 176-179. doi: 10.4067/ S0717-77122007000200012

6 Arrubla M, Cárdenas R, Posada F. 2008. Adherencia de las esporas de Beauveria bassiana formuladas en polvo y líquido sobre la broca del café. Rev U.D.C.A Actualidad \& Divulgación Científica 11: 123-133.

7 Bastidas A, Velásquez E, Marín P, Benavides P, Bustillo A, Orozco F. 2009. Evaluación de preformulados de Beauveria bassiana (Bálsamo) Vuillemin, para el control de la broca del café. Agronomia 17: 44-61.

8 Becerra M, Otrando D. 2020. Keds, las enigmáticas moscas y su papel como vectores de patógenos. Acta Trop 209: 105-521.

9 Cañedo V, Ames T. 2004. Manual de laboratorio para el manejo de hongos entomopatógenos. Lima, Perú: Centro Internacional de la Papa (CIP). 62 p.

10 Chase MR, Raina K, Bruno J, Sugumaran M. 2000. Purification, characterization and molecular cloning of prophenoloxidases from Sarcophaga bullata. Insect Biochem Molec 30: 953967. doi: 10.1016/s0965-1748(00)00068-0

11 Chávez E, Rodríguez S, Sánchez L, Hamdan A, Barranco J. 2014. Actividad insecticida in vitro de extracto crudo de Beauveria bassiana (Bálsamo) Vuillemin sobre larvas de Phyllophaga spp. (Harris). Rev Prot Veg 29: 226-230.

12 Delgado P, Murcia B. 2011. Hongos entomopatógenos como alternativa para el control biológico de plagas. AmbiAgua 6: 77-90. doi: 10.4136/ambiagua. 187

13 Duan D, Liu G, Cheng T, Wang Y. 2017. Microbial population analysis of the midgut of Melophagus ovinus via highthroughput sequencing. Parasite Vector 10: 382. doi: 10.1186/s13071-017-2323-1

14 Feijó F, Lima P, Alves N. Luna E. 2007. Comportamento e aspectos citológicos de Beauveria basssiana após passagem em ovo, larva e adulto de Chrysomya albiceps. Arq Inst Biol 74:349-355.
15 Gómez R, Nova A, Méndez P, Palazuelos P, Venegas R. 2012. Manual. Control biológico de Melophagus ovinus. Chile: Centro de Educación y Tecnología, Ministerio de agricultura. $28 \mathrm{p}$.

16 Harrison R, Bonning B. 2010. Proteases as insecticidal agents. Toxins 2: 935-953. doi: 10.3390/toxins2050935

17 Holdsworth PA, Vercruysse J, Rehbein $S$, Peter RJ, De Bruin C, Letonja T, Green P. 2006. World Association for the Advancement of Veterinary Parasitology (W.A.A.V.P.) guidelines for evaluating the efficacy of ectoparasiticides against biting and nuisance flies on ruminants. Vet Parasitol 136: 3-13. doi: 10.1016/j.vetpar.2005.11.006

18 Jiang, U, Yuan U, Yang R, Zhang $N$, Gu N. 2019. Beauveria bassiana is a potential effective biological agent against Psoroptes ovis var. cuniculi mites. Biol Control 131: 43-48

19 Khan S, Guo I, Maimaiti Y, Mijit M, Qiu D. 2012. Entomopathogenic fungi as microbial biocontrol agent. Molecular Plant Breed Biotechnol 3: 63-79.

20 Kirkland B, Eisa A, Keyhani N. 2005. Oxalic acid as a fungal acaracidal virulence factor. J Med Entomol 42: 346351. doi: 10.1093/jmedent/42.3.346

21 Larroza M, Leiva D, Escribano C, Disalvo V, Montero V, Pantoja C, Olmedo E, et al. 2012. Caracterización de la melofagosis en la provincia de Tierra del Fuego, Argentina. $35^{\circ}$ Congreso Argentino de Producción Animal. Córdoba, Argentina.

22 Larroza M. 2013. Caracterización de la melofagosis en ovinos en la Región Patagónica: ciclobiológico, dinámica poblacional y distribución. Tesis Doctoral.Argentina: Univ. Nacional de La Plata. 118 p.

23 Manosalva L, Oyarzun A, Freile ML, Quiroz A, Fajardo V. 2011. Promissory insecticide activity of compounds of plants against sheep keds (Melophagus ovinus) (Dipetero: Hippoboscidae), sheep ectoparasite. $3^{\text {rd }}$ International Workshop: Advances in Science and Technology of Biosource. Pucón, Chile. 
24 Mejía OS, Gómez RJ, López GG, Cruz LL, Valle MJ, 2016. Evaluation of the pathogenicity of isolates of Beauveria bassiana against Rhyssomatus nigerrimus. Southwest Entomol 41: 41-50. doi: 10.3958/059.041.0106

25 Monzón A. 2001. Producción, uso y control de calidad de hongos entomopatógenos en Nicaragua. Manejo Integrado de Plagas 63: 95-103.

26 Nelson WA, Bainborough AR. 1963. Development in sheep of resistance to the ked Melophagus ovinus (L). III. Histopathology of sheep skin as a clue to the nature of resistance. Exp Parasitol 13: 118-127. doi: 10.1016/00144894(63)90061-4

27 Nelson WA, Kozub GC. 1980. Melophagus ovinus (Diptera: Hippoboscidae): evidence of local mediation in acquired resistance of sheep to keds. J Med Entomol 17: 291-297. doi: 10.1093/ jmedent/17.4.291

28 Obando JA, Bustillo AE. 2011. Selección de cepas de Metarhizium anisopliae para el control de Aeneolamia varia (Hemiptera: Cercopidae). Rev Colomb Entomol 39: 26-33.

29 Olaechea F, Corley J, Larroza M, Raffo F, Cabrera R. 2006. Ingreso y evolución del parasitismo por Melophagus ovinus en una majada Corriedale en el noroeste de la Patagonia Argentina. Parasitol Latinoam 61: 86-89. doi: 10.4067/S0717-77122006000100013

30 Olaechea F. 2009. Aspectos básicos del ectoparásito más frecuente que afecta las majadas del sur patagónico, Malófagos ovinus. Veterinarios Patagónicos 554: 5-7.

31 Ortiz-Urquiza A, Keyhani NO. 2013. Action on the surface: entomopathogenic fungi versus the insect cuticle. Insects 4: 357-374. doi: 10.3390/insects4030357

32 Pedrini N, Crespo R, Juárez P. 2007. Biochemistry of insect epicuticle degradation by entomopathogenic fungi. Comp
Biochem Phys C 146: 124-137. doi: 10.1016/j.cbpc.2006.08.003

33 Pucheta M, Flores A, Rodriguez S, De La Torre M. 2006. Mecanismo de acción de los hongos entomopatógenos. Rev Mex Micol 31: 856-860.

34 Rao G, Narladkar B. 2018. Role of entomopathogenic fungi in tick control: a review. J Entomol Zool Stud 6: 12651269.

35 Scasta JD, Koepke, K. 2016. Hostparasite ecology of keds (Melophagus ovinus (L)) relative to sheep breed and age on Wyoming rangeland. Livest Sci 189: 17-22. doi: 10.1016/j.livsci.2016.05.002

36 Small RS. 2005. A review of Melophagus ovinus (L), the sheep ked. Vet Parasitol 130: 141-155. doi: 10.1016/ j.vetpar.2005.03.005

37 Téllez A, Cruz M, Mercado Y, Torres A, Cuenca A. 2009. Mecanismos de acción y respuesta en la relación de hongos entomopatógenos e insectos. Rev Mex Micol 30: 73-80.

38 Tofiño A, Ortega M, Pedraza B, Perdomo S, Moya D. 2018. Efectividad de Beauveria bassiana (Baubassil@) sobre la garrapata común del ganado bovino Rhipicephalus microplus en el Departamento de la Guajira, Colombia. Rev Argent Microbiol 50: 426430. doi: 10.1016/j.ram.2017.10.005

39 Vargas-Albores F, Ortega-Rubio A. 1994. El sistema immune humoral de los insectos. Tópicos de Investigación y Posgrado 4: 21-28.

40 Wang C, St Leger RJ. 2007. The MAD1 adhesin of Metarhizium anisopliae links adhesion with blastospore production and virulence to insects, and the MAD2 adhesin enables attachment to plants. Eukaryot Cell 6: 808-816. doi: 10.1128/EC.00409-06 